\title{
Agent-based modelling of consumer purchased behavior considering website quality
}

\author{
Nan Zhang \\ Harbin University of Commerce, Harbin 150028, China \\ zhangnan@hrbcu.edu.cn
}

Keywords: Agent-based model; Consumer purchased behavior; Website Quality

\begin{abstract}
The problem of consumer purchase behaviour has been concerned by many researchers. The paper presents an agent-based model of consumer purchased behaviour. The main of agentbased modelling is the modifier motivation function that considered price of commodities, quality of commodities, and website quality. Moreover, the study used Repast Simphony toolkit to simulate the modifier motivation function. The method of agent-based simulation gave a dynamic expression in consumer purchased behaviour.
\end{abstract}

\section{Introduction}

Agent-based modelling is a form of computational social science, which is a new analytical and computational method [1-3]. Agent-based modelling has offered an interesting method to solve some complex issues in many fields of study [4-5]. Jin Chun et al. [6] introduced an agent-based modelling and simulation method to build the relationship between customer behaviour and personalized recommendation. Zhao Aiwu et al. [7] described green purchase behaviour of bounded rational consumers based on computational experiments. Jin Chun et al. [8] proposed an agentbased modelling and simulation method on the consumer's purchasing behaviours under the website promotions. Schenk et al. [9] presented the particulars of an agent-based micro model for grocery shopping, based on an individual population and store data gathered in northern Sweden. Roozmand et al. [10] proposed an agent-based conceptual and computational model of consumer decisionmaking based on culture, personality and human needs. Du Jianguo et al. [11] established an agentbased computational experiments model for evolution of enterprise's productive behaviour of green products.

With the development of e-commerce, consumer purchase behaviour is concerned by many scholars. In this paper, consumer purchase behaviour is online shopping of consumer behaviour. In general, consumer purchased behaviour includes five stages: need recognition, information search, evaluation of alternatives, purchase decision, and post-purchase behaviour [12]. Need recognition is also called problem identification, which is the first step in consumer purchased behaviour. It occurs when a consumer discovers an unmet need that must be fulfilled. Information search is a consumer searches for internal or external information. Evaluation of alternatives is a stage, in which consumers compare the brands and products that are in their evoked set. Purchase decision is a stage, where the purchase takes place. Post-purchase behaviour is when the customer assesses whether he is satisfied or dissatisfied with a purchase. Consumer purchase behaviour includes research in the field of personality, psychology, sociology, marketing, which is affected some factors, such as price of commodity, quality of commodity, advertisement and follower tendency et al. [13].

Recently, researchers have confirmed that website quality influence online shopping of the consumer behaviour [14-16]. Yang et al. [17] presented the differences between multinational and domestic e-commerce companies in the influence of website quality on online purchase intention. Li Junjun and Sun Jianjun [18] proposed the user technology adoption model and hypotheses by combining the e-commerce website quality and perception factors. Yang Zhaojun and Song Xueting [19] studied the impacts of different dimensions of website quality and website customer service on 
customer satisfaction and purchase intention by collecting 257 questionnaires.

From these researches above mentioned, the method of mathematics and statistics are used to investigate consumer purchased behaviour considered the website quality. The method means that consumer purchased behaviour is a static process. However, in fact, consumer purchased behaviour is a various and dynastic process. In this paper, we present agent-based modelling of consumer purchased behaviour considering website quality. The remainder of this paper will provide an overview of agent-based modelling, modify the motivation function, simulate the results, and end with conclusions.

\section{Overview of agent-based modelling}

Agent-based modelling enables a researcher to create, analyze, and experiment with models consist of agents that interact within an environments [20]. The agents include autonomy, social ability, responsiveness and active ability [20]. Moreover, agents can pass messages to each other and influence each other by learn from experiences. The major feature of agent-based modelling is that system structures may emerge at the macro-level due to the rules of behaviour at the microlevel for the agents.

Agent-based modelling is a simulation modelling approach that explores and analyzes the dynamic complex systems. The applications range from agent-based modelling in the urban, supply chains, consumer markets and electricity market [20]. Thomas Schelling [21] proposed a dynamic model of segregation to state phenomenon of residential segregation in American cities. The dynamic model of segregation discussed the relationship between individual agent behaviour preference and overall segregation. Zhang Tao and David Zhang [22] presented an agent-based modelling of consumer purchase decision-making to exhibit the emergent decoy effect phenomenon. North et al. [23] built an agent-based modelling of electricity markets to provide a certain quantity of electricity at a certain price for a period. Strader et al. [20] described a model to study the impact of information sharing in divergent assembly supply chains.

Compared to other models, the implementation of agent-based modelling is relatively easy and simple. SWARM, NetLogo and Repast are used to program the agent-based modelling. Furthermore, these tools are freely available. In this paper, we use Repast Simphony to build agentbased modelling. Repast Simphony is a widely used, free, and open source agent-based modelling and simulation toolkit with three released platforms, namely Repast for Java, Repast for the Microsoft .NET framework, and Repast for Python Scripting [24].

\section{The motivation function}

Zhang Tao and David Zhang [22] calibrated the motivation function to computationally simulate the decoy effect. In the paper, Zhang Tao and David Zhang considered that price of commodity, quality of commodity, advertisement and follower tendency influence the consumer purchased behaviour. So, the motivation function was presented as follow:

$$
M_{i}=P S_{i} * P_{i}+Q S_{i} * Q_{i}+s u s_{i} * a d_{i}+f t_{i} * i n f l_{i}
$$

Where $M_{i}$ is the motive function, $P S_{i}$ is price sensitivity parameter, $P_{i}$ is price of commodity, $Q S_{i}$ is quality sensitivity parameter, $Q_{i}$ is quality of commodity, sus $_{i}$ is susceptibility parameter, $a d_{i}$ is the advertising intensity, $f t_{i}$ is follower tendency parameter, and $i n f l_{i}$ is perceived influence.

Consumer purchase behaviour depends on some other variables except for price and quality of commodities. According to the above analyzed, we can see that website quality influences online shopping of the consumer purchased behaviour. Therefore, website quality is put into the motivation function in this paper. Website quality is multi-dimensional variable, which involves information quality, system quality and server quality [13-14]. Here, website quality as shown:

$$
W Q_{i}=\sum_{k=1}^{3} W_{k} Q_{i k}
$$

Where $W Q_{i}$ is website quality, $W_{k}$ is weight of attribute $k, Q_{\mathrm{ik}}$ is quality $k$ 
Therefore, the motivation function is

$$
M_{i}=P S_{i} * P_{i}+Q S_{i} * Q_{i}+s u s_{i} * W Q_{i}
$$

According to the Kim's research, $P S_{i}$ may be expressed as follow:

$$
P S_{i}=\left(-\alpha^{P_{i}-\frac{1}{N} \sum_{i=1}^{N} P_{i}}+k\right) * P_{i}
$$

Meanwhile, Patel and Schlijper [25] indicated that the quality sensitivity parameter of commodity lied on the different value between the real quality of commodity and the expected quality of commodity. $Q S_{i}$ may be expressed as follow:

$$
Q S_{i}=\left(\beta^{\left|Q_{i}-\sum_{j=1}^{m} W_{j} Q_{i j}\right|}+l\right) * Q_{i}
$$

Where $Q_{i j}$ is the quality $j$ of commodity $i$.

Substituting Equation (2), equation (4) and equation (5) into equation (3), the motivation function is modified as:

$$
\begin{aligned}
M_{i} & =P S_{i} * P_{i}+Q S_{i} * Q_{i}+\text { sus }_{i} * W Q_{i} \\
& =\left(-\alpha^{P_{i}-\frac{1}{N} \sum_{i=1}^{N} P_{i}}+k\right) * P_{i}+\left(\beta^{\left|Q_{i}-\sum_{j=1}^{m} W_{j} Q_{i j}\right|}+l\right) * Q_{i}+\gamma * \sum_{k=1}^{3} W_{k} Q_{i k}
\end{aligned}
$$

Where $\alpha, \beta, \gamma, k$ and $l$ are parameters.

\section{Simulation and experiment results}

This paper uses Repast Simphony toolkit to simulate the modified motivation function. Here, we choose three commodities to simulate the motivation function, that is, $\mathrm{i}=3$. The random distribution of the prices and qualities of commodity $i$ are between 0 and 30, between 30 and 60, between 60 and 100. $l$ and $k$ depend on the agent income. Income is random normal distribution. Namely, mean is 500 and variance is $60 . W_{k}$ is random normal distribution, namely, mean is 50 and variance is 20 . Other parameters depend on the training and testing. Table 1 shows the scales and distributions of parameters.

Table 1 the scale and distribution of parameters

\begin{tabular}{|c|c|c|}
\hline Parameter & Scale & Distribution \\
\hline $\mathrm{P}_{1}$ & $0-30$ & Random distribution \\
\hline $\mathrm{P}_{2}$ & $30-60$ & Random distribution \\
\hline $\mathrm{P}_{3}$ & $60-100$ & Random distribution \\
\hline $\mathrm{Q}_{1}$ & $0-30$ & Random distribution \\
\hline $\mathrm{Q}_{2}$ & $30-60$ & Random distribution \\
\hline $\mathrm{Q}_{3}$ & $60-100$ & Random distribution \\
\hline $\mathrm{k}$ & $-100-0$ & Random normal distribution \\
\hline $\mathrm{l}$ & $0-100$ & Random normal distribution \\
\hline $\mathrm{w}$ & $0-100$ & \\
\hline
\end{tabular}

\section{Conclusions}

With the development of e-commerce, online shopping is convenient for consumers. Meanwhile, merchants can also bring long-term benefits. Website quality is clearly critical in driving traffic, making people stay, and eventually attracting people to purchase online products. This study has developed an agent-based model of the impact of website quality on customer purchased behaviour. Moreover, we used Repast Simphony toolkit to simulate the model. Like other research efforts, this study is subject to some limitations, and future studies should relax these limitations. 


\section{Acknowledgements}

The authors would like to thank the constructive comments offered by the anonymous reviewers. This research was supported by the Heilongjiang Postdoctoral Grant No.LBH-Z15115, and the Harbin University of Commerce under Grant No.15RW21.

\section{References}

[1] Sheng Zhaohan, Zhang Wei. Computational experiments in management science and research. Journal of Management Sciences in China, 14(5), 2011, 1-10

[2] Cui Kainan, Zheng Xiaolong, Wen Ding, Zhao Xueliang. Researches and Applications of Computational Experiments. Acta Automatica Sinica, 39(8), 2013, 1157-1169

[3] Chen Yingwu, Xing Lining, Wang Hui, Ge Bin, Li Pei, Zhao Qingsong. Review on Organizational Behaviour Modelling and Computational Experiments of Social Management under Network Environments. Acta Automatica Sinica, 41(3), 2015, 462-470.

[4]Liu Dan, Cao Jiantong, Chen Jianming. Application of Agent-based Modelling Literature Review. Technology Economics, 33(11), 2014, 96-102.

[5] Zhang Wei, Feng Xu, Xiong Xiong. Agent-based Computational Finance in China Its Present State and Future Prospects. Journal of Systems \& Management, 21(6), 2012, 756-764.

[6] Jin Chun, Zhang Yiping. Agent-based simulation model of customer behaviour and personalized recommendation. Systems Engineering-Theory and Practice. 33(2), 2013, 463-472

[7] Zhao Aiwu, Du Jianguo, Guan Hongjun. Green purchase behaviour of bounded rational consumers based on computational experiments. Systems Engineering-Theory and Practice. 35(1), 2015, 95-102

[8] Jin Chun, Dong Qiu, Lu Miao. Agent-based simulation for consumer behaviour under website promotion. Systems Engineering-Theory and Practice. 34(4), 2014, 845-853

[9] Schenk T A, Lo ffler G, Rauh J. Agent-based simulation of consumer behaviour in grocery shopping on a regional level. Journal of Business Research, 60 (8), 2007, 894-903.

[10] Roozmand O, Nasser G A, Hofstede G J, et al. Agent-based modelling of consumer decision making process based on power distance and personality. Knowledge-Based Systems, 24, 2011, 1075-1095.

[11] Du Jianguo, Yu Xiaohui, Meng Qingfeng. Evolution of Enterprise's Productive Behaviour of Green Products Based on Computational Experiments. Soft Science, 30 (11), 2016, 66-70

[12] Engel JF, Blackwell RD, Miniard PW. Consumer Behaviour. 8th ed. Philadelphia: The Dryden Press; 1995

[13] Su Qin, Li Zhao, Cui Yanwu, Chen Ting. An Empirical Analysis on the Antecedents of Internet Customer Behavior. Systems Engineering, 25(2), 2007, 2-6

[14] Zou Rui. The Impact of B2C Website Quality on Customer Attitude Based on TAM Theory Taking Large-scale Comprehensive Websites as Examples.

[15] Chen Yibing. The Impact of E-Commerce Website Quality on Customers online Trust. 2010.

[16] Song Xueting. Research on the Relationship of B2C E-commerce Websites’ Overall Quality, Customer Satisfaction and Purchase Intention. 2013

[17] Yang Zhaojun, Cao Lisha, Zhang Yali. Formative Website Quality and Purchase Intention Cross-validation Analysis of Multinational and Domestic E-commerce Companies. Journal of Modem Information. 35(5), 2015, 41-62 
[18] Li Junjun, Sun Jianjun. Empirical Study on Website Quality, User Perception and Technology Adoption Behaviour. Journal of the China Society for Scientific and Technical Information. 28(3), 2011, 227 - 236.

[19] Yang Zhaojun. Song Xueting. The Impact of Website Quality Dimensions on Customer Satisfaction and Purchase Intention. Journal of Modem Information. 34(2), 2014, 13-17

[20] Gilbert N., Agent-Based Models, Sage publication, 2008

[21] Schelling, T. Dynamic Models of Segregation. Journal of Mathematical Sociology, 1971, 1, 143-186

[22] Zhang T. Zhang D. Agent-based simulation of consumer purchase decision-making and the decoy effect. Journal of Business Research, 60(8), 2007, 912-922.

[23] North M., Thimmapuram P., Cirillo R., et al. EMCAS: An Agent-based Tool for Modelling Electricity Markets. Proceedings of Agent 2003: Challenges in Social Simulation.

[24] North M.J., Collier N.T., and Vos J.R.. Experiences Creating Three Implementations of the Repast Agent Modelling Toolkit. ACM Transactions on Modelling and Computer Simulation, 16(1), 2006, 125

[25] Patel S. Schlijper A. Model of consumer behaviour [R]. Report on ESGI 2004, 2004. 